\title{
An integrated modelling approach for R5-X4 mutation and HAART therapy assessment
}

\author{
Anil Sorathiya $^{1}$. Andrea Bracciali ${ }^{2}$. \\ Pietro Liò ${ }^{1}$
}

Received: date / Accepted: date

\begin{abstract}
We have modelled the within-patient evolutionary process during HIV infection using different methodologies. New viral strains arise during the course of HIV infection. These multiple strains of the virus are able to use different coreceptors, in particular the CCR5 and the CXCR4 (R5 and X4 phenotypes, respectively) influence the progression of the disease to the AIDS phase. We present a model of HIV early infection and CTLs response which describes the dynamics of R5 quasispecies, specifying the R5 to X4 switch and effects of immune response. We illustrate dynamics of HIV multiple strains in the presence of multidrug HAART therapy. The HAART combined with X4 strain blocker drugs might help to reduce infectivity and lead to slower progression of disease. On the methodology side, our model represents a paradigm of integrating formal methods and mathematical models as a general framework to study HIV multiple strains during disease progression, and will inch towards providing help in selecting among vaccines and drug therapies. The results presented here are one of the rare cases of methodological cross comparison (stochastic and deterministic) and a novel implementation of model checking in therapy validation.
\end{abstract}

Keywords HIV · HIV dynamics · HAART · deterministic modelling · stochastic modelling $\cdot$ model checking

This project is supported by EC IST SOCIALNETS - Grant agreement number 217141. Andrea Bracciali has been supported by an EMBO Short Term Fellowship.

\footnotetext{
${ }^{1}$ A. Sorathiya and P. Liò

Computer Laboratory, Cambridge University, William Gates Building, 15 JJ Thomson Avenue, Cambridge CB3 0FD, UK

E-mail: \{anilkumar.sorathiya, pietro.lio\}@cl.cam.ac.uk

${ }^{2}$ A. Bracciali

Department of Computing Science and Mathematics, University of Stirling, UK

E-mail: braccia@cs.stir.ac.uk

(formerly Computer Science Department, University of Pisa, Largo Bruno Pontecorvo, 3, Pisa 56127, IT)
} 


\section{Introduction}

The study of infectious diseases which underlie the immune system response to pathogens, at both levels of cellular response (where parameters include genomic and metabolic factors) and population of individuals (where parameters include age, structure and social networks), has received a major impetus from the recent development of high-throughput genomic techniques. The use of computational system biology models in immunology has been very successful and has represented an insightful and essential complement to in-vivo and in-vitro experimental design and interpretation. Indeed computational system biology models of HIV dynamics have proven valuable in understanding the mechanisms of many of the observed features of the progression of the HIV infection, see for example (Celada and Seiden, 1996; Chao et al., 2004; De Boer and Perelson, 1995; Ho et al., 1995; Perelson et al., 1996; Wei et al., 1995; Wiegel and Perelson, 2004; Wodarz and Nowak, 2002).

Here, we address the issue of formally modelling and studying the dynamics of HIV infection with the long term goal of engineering treatments that may sustain the immune response to the disease. We present a model of HIV early infection which describes the dynamics of R5 quasispecies, specifying the R5 to $\mathrm{X} 4$ switch and effect of the immune response. Furthermore, HAART therapy has been embedded in the model, and its effects analysed on the progression of the disease. For clarity, a summary of the most relevant aspects in this scenario is given next, separately.

Importantly, we illustrate an approach consisting of the integration of diverse methodologies from deterministic models traditionally employed, to stochastic descriptions of such a dynamics, whose application to this context is quite novel (Sorathiya et al., 2010). Furthermore, we illustrate the use of stochastic model checking, a relevant formal reasoning technique developed in computer science, on the results obtained from the simulation of such stochastic models.

Model checking consists in specifying a property of interest, such as the average reduction of infected cells or pathogen density in the blood as consequence of the (regular or salutary) assumption of a given drug, and then verifying whether this property holds for the system at hand. The property is expressed in a formal language, typically a formula of a suitable logic, and its verification is carried out by a fully automated procedure that returns either a positive response or a counter example, such as a working hypotheses that is able to annul the effects of the drug under investigation. Given that viral infections and the functioning of therapies often present stochastic aspects, we have introduced a succinct but descriptive stochastic model of HIV infection and then extended it by modelling (the effects of) anti-HIV therapies. The model has been implemented in PRISM, a state of the art probabilistic model checker, supporting a logical language.

The aim of this paper is to illustrate how the use of quantitative models of infectious processes can further be enhanced by the contribution of formal methods originally developed for verifying properties of computational systems. In particular, we illustrate how model checking techniques (Clarke et al., 1999) can contribute to the interpretation of the in-silico results obtained from the quantitative models. We envisage that this will provide a valuable methodology potentially scaling up to assess and develop therapies for infectious disease treatments, helping in bridging the gap between theoretical and experimental investigation and the 
medical practice (i.e. translational medicine). The results and the methodology discussed here suggest the importance of devising formal analysis and assessment techniques from the integration, and extension, of modelling and reasoning techniques developed in different contexts.

Next Section 2 gives an account of a general scenario of HIV infection. A deterministic model based on ODEs and a stochastic one based on a framework from theoretical computer science are described in Section 3. In Section 4 we discuss therapies which might be helpful in medication of HIV-1 infected patients. In addition, immune response models may help in designing vaccines. Most importantly, we report on in-silico results from different techniques, as an example of integrated framework suitable for modelling and reasoning about infection dynamics and immune response.

\section{Biological context}

HIV-1 infection is characterised by the progressive loss of CD4 + T cells. Infection by most strains of HIV-1 requires interaction with CD4+ $\mathrm{T}$ cell and a chemokine receptor, either CXCR4 or CCR5. Switching of CCR5 (R5) to CXCR4 (X4) has been linked to an increased virulence and with progression to AIDS, probably through the formation of cell syncytia and killing the $\mathrm{T}$ cell precursors. CXCR4 is expressed on a majority of CD4+ T cells and thymocytes, whereas only about 5 to $25 \%$ of mature $\mathrm{T}$ cells and 1 to $5 \%$ of thymocytes express detectable levels of CCR5 on the cell surface (Gray et al., 2005). It is noteworthy that X4 HIV1 strains stimulate the production of the cellular factor called Tumor Necrosis Factor (TNF), which is associated with immune hyperstimulation, a state often implicated in T-cell depletion (Herbeuval et al., 2005). TNF seems to be able to both inhibit the replication of R5 HIV strains while having no effect on X4 HIV and down regulate the number of CCR5 co-receptors that appear on the surface of T-cells (PY2).

Cytotoxic T Lymphocytes (CTLs) play a vital role in controlling infection and variability of HIV-1. The pressure on HIV-1 progression is the existence of either weak CTL selection pressure or viral mutations (Carrington and OBrien, 2003). A recent study shows that higher degree of immunodominance leads to more frequent escape with a reduced control of viral replication but a substantially impaired replicative capacity of the virus (Althaus and Boer, 2008). We aim at modelling viral multi-strain short and long term evolutionary dynamics especially transition of R5 to X4 phenotype switch and CTLs response.

In the next section we introduce a multiple strains model focusing on the R5 to X4 shift, the hyperstimulation of T cell precursors through TNF (Sguanci et al., 2007) and the CTLs response. We describe the decreasing dynamics of CD4+ T cells after the appearance of X4 strains and make predictions on the results of HAART.

\section{Models}

We have developed a multi-strain computational model of HIV-1 viral dynamics including a cellular response of CTLs. Infected $\mathrm{T}$ cells mutate from one type of 


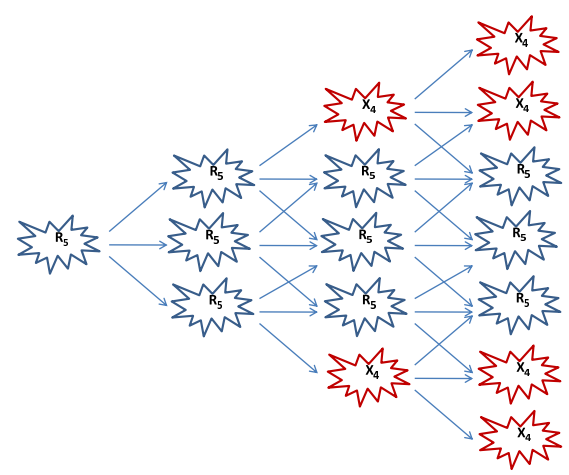

Fig. 1 Schematic representation of R5 to X4 phenotype mutation. R5 strain mutates and proliferates into other R5 strains, and in the later stage of disease, R5 mutation leads to $\mathrm{X} 4$ strains. Each stage of mutation of R5 or X4 has the possibility to generate another R5 strain/X4 strain.

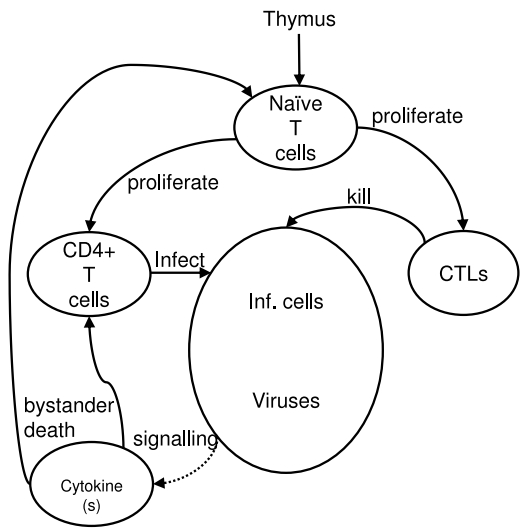

Fig. 2 Schematic description of interaction among the cells and viruses. Circle $\bigcirc$ represents population of cells/virons, $\rightarrow$ abundance of cells/viral particles from one type to the other, either directly from single cell population or through interaction of two different type of cell populations. The interaction among Naive T cells (U), uninfected mature $T$ cells $(T)$, infected cells (I), viral strains $(\mathrm{V})$, TNF (cytokine $-\mathrm{F}$ ), and CTL response $(\mathrm{Z})$ is translated in the ordinary differential equations in the Models section.

strain (R5) to another type of strain (X4) at the rate $\mu$. Mutation parameter $\mu$ generates additional strains of virus from existing phenotype strains. The graphical representation of this process is given in Figure 1. For sake of clarity, we have provided a schematic description of the interaction occurring between various kind of cells (see Figure 2). Here, we have considered $k$ different strains of CTLs, Viral particles and Infected cells. Naive $\mathrm{T}$ cells are continuously produced from the thymus. The mature T cells and CTLs cells proliferate from naive T cells, in which CD4+ T cells are infected by viral particles. Mutation of viruses inside infected cells produces different strains of viral particles. Viral particles mutate from R5 strains to X4 strains by mutation rate $\mu$. We have included TNF cytokine which 
induces "bystander death" of both naive and mature cells. Detailed description of each type of cell and viral particles has been described in section 3.1.

\subsection{The deterministic model}

Our work is based on the evolution of a multi-strain model of HIV-1 dynamics, first appearing in (Sguanci et al., 2007), which takes into account the models developed by Perelson and his followers (Chao et al., 2004; Ho et al., 1995; Perelson et al., 1996; Wiegel and Perelson, 2004). These models are well presented and take specific biological reality into account. The studies have shown that the equilibrium abundance of the infected cells depends on the immune response (Cannon et al., 1988; Riddell et al., 1992). Therefore, our initial model has been extended here by adding the response of Cytotoxic T Lymphocytes (CTLs) cells.

As shown in several studies, CTLs play an important role in controlling HIV infection especially at the initial stage (Kaslow et al., 1996; Schmitz et al., 1999). However, there are many challenges to model immune response because HIV is difficult to diagnose in the early stage. Additionally, to acquire data on CTLs escape is difficult since the virus diversity within a host has to be followed over a long time. It is also difficult to analyse the intersection in viral replication, the selection and the mutation by different CTLs response (Fryer et al., 2009; Ganusov and De Boer, 2005). To better understand these processes, we present the multistrain deterministic model and the extensions regarding therapies.

Model description The interactions among the immature T cells (U), uninfected mature T cells (T), CTL response (Z), infected cells (I), viral strains (V), and TNF (F) are translated into ordinary differential equations (ODEs). The changes in the population over time can be described by following ordinary differential equations:

$$
\begin{aligned}
\frac{d U}{d t} & =\lambda+\sigma_{1}\left(\sum_{k} I_{k} Z_{k}\right)-\delta_{u t} U-\delta_{u f} U F-\delta_{u z} U \\
\frac{d T}{d t} & =\delta_{u t} U-\left(\sum_{k \in R 5} \beta_{k 5} V_{k}+\sum_{k \in X 4} \beta_{k 4} V_{k}\right) T-\delta_{t f} T F-\delta_{t} T \\
\frac{d Z_{k}}{d t} & =\delta_{u z} U+\sigma_{2} I_{k}-\delta_{z} Z_{k} \\
\frac{d I_{k}}{d t} & =\left(\sum_{k^{\prime}} \mu_{k k^{\prime}} \beta_{k^{\prime}} V_{k^{\prime}}\right) T-\delta_{i z} I_{k} Z_{k}-\delta_{i} I_{k} \\
\frac{d V_{k}}{d t} & =\pi I_{k}-\delta_{v} V_{k} \\
\frac{d F}{d t} & =\sigma_{3} \sum_{k \in X 4} V_{k}
\end{aligned}
$$

Equation (1) describes the constant production of immature $\mathrm{T}$ cells by the thymus at rate $\lambda$ and their transformation into mature T cells and CTLs cells at rate $\delta_{u t}$ and $\delta_{u z}$ respectively. If X 4 viruses are present, upon the interaction with TNF, immature T-cells are cleared at fixed rate $\delta_{u f}$. Moreover, immature T cells 
proliferate, due to CTLs via dendritic cells signaling, at fixed rate $\sigma_{1}$. To simplify model, we have modelled direct effect of CTLs on the immature T cells instead of adding one more equation of dendritic cells signal to naive $\mathrm{T}$ cells for optimal activation (Guermonprez et al., 2002).

Equation (2) describes how uninfected mature $\mathrm{T}$ cells are produced at fixed rate $\delta_{u t}$ by the pool of immature T cells. These cells interact with any strain of the virus, $V_{k}$ and become infected at rate $\beta_{k}=\beta \quad \forall k$. The infectiousness parameter, $\beta$, is not constant over time, but depends on the interplay between R5 and X4 viruses. In particular, due to the presence of TNF, the infectivity of the R5 strains reduces $\left(\beta_{R 5}(t)=\beta-k_{R 5} F(t)\right.$, with constraint $\left.\beta_{R 5} \geq 0\right)$, while infectivity of the $\mathrm{X} 4$ strains increases $\left(\beta_{X 4}(t)=\beta+k_{X 4} F(t)\right)$.

Equation (3), models CTLs strains which are produced at fix rate $\delta_{u z}$ from pool of immature $\mathrm{T}$ cells. In addition, these cells proliferate due to infectivity at rate $\sigma_{2}$. CTLs cells are cleared out at fixed rate $\delta_{z}$.

Equation (4) describes the infection of mature CD4+ T cells. Infected cells of strain $k$ arise upon the interaction of a virus of strain $k$ with any of the mature T-cell strains. The infected cells, in turn, are cleared out at rate $\delta_{i}$. In addition, the infected cells are cleared out by CTLs response at fixed rate $\delta_{i z}$.

Equation (5) describes the production of viral strains from infected cells at fixed rate $\pi$, viruses are cleared out at fixed rate $\delta_{v}$.

Equation (6) models the dynamics of accumulation of TNF by assuming the increase in TNF level to be proportional, via the constant $\sigma_{3}$, to the total concentration of X4 viruses present.

All the parameters have been referred from literatures. The detailed description and references of each parameters are given in the Table 3.2.

Highly Active Antiretroviral Therapy (HAART) HAART is a cocktail of drugs prescribed to many HIV-positive patients. HAART is one of the ways of suppressing viral replication in the blood while attempting to prevent the virus rapidly developing resistance to the individual drug. The therapy is composed of usually three combinations of drugs; one nucleoside analog (DNA chain terminator), one protease inhibitor and either a second nucleoside analog ("nuke") or a nonnucleoside reverse transcription inhibitor (NNRTI) (Dybul et al., 2002). Here, we have developed a model for non-nucleoside reverse transcription inhibitor (also known as RT inhibitor) and protease inhibitor drugs in the HAART therapy. According to guidelines, it is important to take every dose of medication as prescribed. Poor adherence to HAART has been shown to result in treatment failure, and faster disease progression. If doses are left out, the virus can replicate faster and the risk of developing drug resistance rises. We have observed this phenomenon in the previous work (Sguanci et al., 2007).

RT Inhibitor: An RT inhibitor drug is usually prescribed to block infection therefore CD4+ T cells do not get infected by viruses. This drug can be modelled by reducing $\beta$. In $100 \%$ working condition of $\mathrm{RT}$ inhibitor, $\beta=0$ means viruses do not infect $\mathrm{T}$ cells. But, RT inhibitors are not perfect like other drugs. Thus, a more realistic model for the effectiveness of an RT inhibitor is described by multiplying $\left(1-\eta_{R T}\right)$ with $\beta$ parameter, where $\eta_{R T}$ is the "effectiveness" of the reverse transcription inhibitor (value: $0 \leq \eta_{R T} \leq 1$ ). The effectiveness of the RT inhibitor can be modelled by changing equation 2 into equation 8 . Similarly, equation 4 changes into equation 10 . 
Protease Inhibitors (PIs) and Nucleoside Analog (DNA chain terminator): Protease inhibitors are a class of drugs used to prevent viral replication by inhibiting the activity of HIV-1 protease. HIV-1 uses their protease as an enzyme to cleave nascent proteins so that new viruses can be produced. The drug, PIs, is used to block the cleave nascent proteins of HIV-1. Similarly, the nucleoside analog acts to prevent production of new viruses by blocking the viral DNA chain during infection of a new cell by HIV-1. The effectiveness of PIs and nucleoside analog can be modelled similarly by reducing the $\pi$ parameter value. The following model describes the effect of PIs, nucleoside analog and RT inhibitors, where $\eta_{P I}$ is the "effectiveness" of the protease inhibitors (value: $0 \leq \eta_{P I} \leq 1$ ). The effectiveness of PIs and nucleoside analog can be adapted by changing equation 5 into equation 11 . We have simulated drug therapies and drug resistance in the results section.

$$
\begin{aligned}
\frac{d U}{d t} & =\lambda+\sigma_{1}\left(\sum_{k} I_{k} Z_{k}\right)-\delta_{u t} U-\delta_{u f} U F-\delta_{u z} U \\
\frac{d T}{d t} & =\delta_{u t} U-\left(\sum_{k \in R 5}\left(1-\eta_{R T}\right) \beta_{k 5} V_{k}+\sum_{k \in X 4}\left(1-\eta_{R T}\right) \beta_{k 4} V_{k}\right) T \\
& -\delta_{t f} T F-\delta_{t} T \\
\frac{d Z_{k}}{d t} & =\delta_{u z} U+\sigma_{2} I_{k}-\delta_{z} Z_{k} \\
\frac{d I_{k}}{d t} & =\left(\sum_{k^{\prime}} \mu_{k k^{\prime}}\left(1-\eta_{R T}\right) \beta_{k^{\prime}} V_{k^{\prime}}\right) T-\delta_{i z} I_{k} Z_{k}-\delta_{i} I_{k} \\
\frac{d V_{k}}{d t} & =\left(1-\eta_{P I}\right) \pi I_{k}-\delta_{v} V_{k} \\
\frac{d F}{d t} & =\sigma_{3} \sum_{k \in X 4} V_{k}
\end{aligned}
$$

\subsection{The stochastic model}

Deterministic models based on differential equation, like the one previously introduced, have long been used for immune system and viral infection modelling. They focus on the average behavior of large populations of perfectly mixed, identical individuals. An improved realism is provided by more complicated models such as adding time delays or age-structured partial differential equations. The role of stochastic fluctuations has recently received a renewed interest (Agrawal et al., 2009; Weinberger and Shenk, 2007), since the focus has moved to mesoscopic scales in which the number of interacting elements are quite small, the noise features are not so trivial (i.e. gaussian) and can drive the system towards unexpected behaviour. In particular, agent-based simulation considers the actions of a large number of simple entities, or agents (viruses and cells), in order to observe their aggregate behaviour (Chao et al., 2004). Each agent consists of state variables and a set of rules that governs its behaviour. These rules cause state transitions and have associated a stochastic rate, representing the probability of the interaction causing the transition to occur. The natural semantics of this model is given in terms of Continuous Time Markov Chains (CTMC), in which the stochastic rates 
associated with each transition can be derived from the kinetic rates of the reactions (Kwiatkowska et al., 2009). We employ a population-based approach where the number of each type of species or cells are modelled, rather than the state of each individual component. Agents can interact either directly with each other or indirectly through the environment. The combined behaviour of these agents is observed in a discrete-time or event-driven simulation. Simulations may require long time and great computational power in order to correctly evaluate parameter averages and fluctuation behaviors of the system. Therefore the two types of approach, differential equations and the stochastic one, have substantial pros and cons and a good degree of complementarity. The availability of both approaches, although time and resources expensive, could result in a versatile, compound analysis technique. Furthermore, in our case the definition of a stochastic model allows the interpretation of the simulation results to be carried out from a model checking perspective.

Model description. The stochastic model we present has been derived from the deterministic one, and it models the same dynamics discussed for that model. On top of these working hypotheses two simplifications have been made: i) We group the behaviour of the viral strains into two classes of individuals: one representing the R5 viral strain $(V 5)$ and the other one representing the $\mathrm{X} 4$ viral strain $(V 4)$, which are the strains we focus on when observing the time course of infections. Henceforth, multi-strain dynamics revert to individuals of the $V 5$ strains that suitably mutate into individuals of the $V 4$ strains. This transition has a given associated rate $\mu$ (see Table 3.2). Basically, analogous to the deterministic model, we have included sufficient details of interaction among the species so as to express the properties of interest. ii) For the sake of computational efficiency, we have reduced the simulation time interval over which we validate the properties of interest by means of approximated model checking. Approximated model checking validates a property against a sample of simulations, instead of considering the whole CTMC associated to the stochastic model. The number of such simulations can be determined according to the desired precision of the analysis. Shortening each simulation makes the process more efficient. In order to do this we individuated steady-state time intervals that could be compressed without loss of information. We have also implemented a notion of time in terms of an agent varying its quantity linearly with the time flux. Basically, we have added a stochastic process that does a "tick" at approximately constant time intervals. In our case the time interval is about 10 days. Accordingly a counter is incremented tracing the passage of time which implements a notion of measurable time flux and other processes have access to this counter, so that a mutation can be "activated" at about a given time instance. This is a, often suitable, modelling assumptions that may simplify the description of time-dependent more complex processes.

Repeated stochastic simulations of the model, also carried out with different tools such as Bio-PEPA (Ciocchetta and Hillston, 2009) and Stochkit (Li et al., 2008), have shown a steady state around a few hundred days and then after a few hundred days after the main R5 to X4 mutation. Hence, we have reduced the $[0-4000]$ days time interval to $[0-1000]$ and triggered the mutation at about day 500. This appears to be a reasonable simplification for the methodological purposes of this paper, and a good compromise as far as computational costs are concerned. 


\begin{tabular}{|c|c|c|c|c|}
\hline Description & Symbol & Value & Units & Comments/References \\
\hline $\begin{array}{l}\text { Constant production of immature } \mathrm{t} \\
\text { cells from thymus }\end{array}$ & $\lambda$ & $200($ range $100-500)$ & $\mathrm{cell} / \mu \mathrm{l} \mathrm{t} t^{-1}$ & (Sguanci et al., 2007) \\
\hline $\begin{array}{l}\text { Production rate of mature } C D 4 T \text { cells } \\
\text { from immature T cells }\end{array}$ & $\delta_{u t}$ & 0.129 & $t^{-1}$ & (Ribeiro et al., 2002a) \\
\hline $\begin{array}{l}\text { Death rate immature } \mathrm{T} \text { cells due } \mathrm{TNF} \\
\text { (Apoptosis) }\end{array}$ & $\delta_{u f}$ & $10^{-5}$ & $\mu \mathrm{l} /$ cell $t^{-1}$ & (Sguanci et al., 2007) \\
\hline $\begin{array}{l}\text { Indirect proliferation of immature } t \\
\text { cells due to Infection }\end{array}$ & $\sigma_{1}$ & 0.0001(range $0.0001-0.01)$ & $\mu l /$ cell $t^{-1}$ & Estimation \\
\hline $\begin{array}{l}\text { Production rate of mature CTLs from } \\
\text { immature } T \text { cells }\end{array}$ & $\delta_{u z}$ & 0.005 & $t^{-1}$ & (Ribeiro et al., 2002a) \\
\hline $\begin{array}{l}\text { Infection rate of CD } 4 \mathrm{~T} \text { cells by HIV-1 } \\
\text { viruses }\end{array}$ & $\beta$ & $4 \times 10^{-5}$ & $\mu l /$ cell $t^{-1}$ & $\begin{array}{l}\text { (Layne et al., 1998; Murray et al., 1998; } \\
\text { Phillips, 1996; Stafford et al., 2000) }\end{array}$ \\
\hline Death rate of $C D 4 T$ cells & $\delta_{t}$ & 0.01 & $t^{-1}$ & $\begin{array}{l}\text { CD4+ T cells has life span on average two } \\
\text { years (Michie et al., 1992; Stafford et al., 2000). }\end{array}$ \\
\hline $\begin{array}{l}\text { Death rate immature } \mathrm{T} \text { cells due TNF } \\
\text { (Apoptosis) }\end{array}$ & $\delta_{t f}$ & $10^{-5}$ & $\mu l /$ cell $t^{-1}$ & $\begin{array}{l}\text { We assume death rate T cells due TNF is same } \\
\text { as death rate immature T cells due TNF }\left(\delta_{u f}\right)\end{array}$ \\
\hline $\begin{array}{l}\text { Proliferation rate of CTLs due to In- } \\
\text { fection }\end{array}$ & $\sigma_{2}$ & 0.001 & $\mu l /$ cell $t^{-1}$ & (Ribeiro et al., 2002b) \\
\hline Death rate of CTLs & $\delta_{z}$ & 0.01 & $t^{-1}$ & (Casazza et al., 2001; Ogg et al., 1999) \\
\hline Death rate of infected $\mathrm{T}$ cells & $\delta_{i}$ & 0.33 & $t^{-1}$ & $\begin{array}{l}\text { (Perelson et al., 1996; Stafford et al., 2000) } \\
\text { In some cases for latently infected cells } \\
\text { range is estimated 0.00052-0.001 (Finzi et al., } \\
\text { 1999; Ramratnam et al., 2000, 2004; Zhang } \\
\text { et al., 1999b). For short-lived infected cells } \\
\text { (0.6) (Perelson et al., 1996) and long lived } \\
\text { death rate is } 0.04 \text { (Stafford et al., 2000). }\end{array}$ \\
\hline $\begin{array}{l}\text { Death rate of infected } C D 4 T \text { cells due } \\
\text { to CTLs }\end{array}$ & $\delta_{i z}$ & 0.06 & $\mu l /$ cell $t^{-1}$ & (Asquith et al., 2006) \\
\hline $\begin{array}{l}\text { Production rate of viruses from a In- } \\
\text { fected cell }\end{array}$ & $\pi$ & 200 & $\mu l /$ cell $t^{-1}$ & (Haase, 1999) \\
\hline Death rate of viruses & $\delta_{v}$ & 3 & $t^{-1}$ & $\begin{array}{l}\text { Life span of HIV- } 1 \text { is } 1 / 2 \text { day to } 1 / 3 \text { day (Perel- } \\
\text { son et al., } 1996 \text {; Stafford et al., 2000). In } \\
\text { some studies have given even higher estima- } \\
\text { tions (Mittler et al., } 1999 \text {; Zhang et al., 1999a) }\end{array}$ \\
\hline Production rate of TNF & $\sigma_{3}$ & 0.0001 & $t^{-1}$ & (Sguanci et al., 2007) \\
\hline Viral mutation rate & $\mu$ & $2.5 \times 10^{-3}$ & & (Sguanci et al., 2007) \\
\hline $\begin{array}{l}\text { Decreasing infectivity of R5 phenotype } \\
\text { due to TNF }\end{array}$ & $K_{R 5}$ & $10^{-7}$ & $(\mu l / c e l l)^{2} t^{-1}$ & (Sguanci et al., 2007) \\
\hline $\begin{array}{l}\text { Increasing infectivity of X4 phenotype } \\
\text { due to TNF }\end{array}$ & $K_{X 4}$ & $10^{-7}$ & $(\mu l / c e l l)^{2} t^{-1}$ & (Sguanci et al., 2007) \\
\hline
\end{tabular}

Table 1 Parameters used in the model: Most of the parameters are from biological literature (Casazza et al., 2001; Haase, 1999; Layne et al., 1998; Mansky and Temin, 1995; Michie et al., 1992; Ogg et al., 1999; Phillips, 1996; Ramratnam et al., 2000, 2004; Ribeiro et al., 2002a; Siliciano et al., 2003; Zhang et al., 1999a,b). Even parameters from the mathematical modelling literatures (Asquith et al., 2006; Mittler et al., 1999; Murray et al., 1998; Perelson et al., 1996; Ribeiro et al., 2002b; Sguanci et al., 2007; Stafford et al., 2000) are estimated from biological experimental studies. 


\begin{tabular}{|c||c||c||c|}
\hline$U_{0} \stackrel{\lambda}{\rightarrow} U_{0}+U$ & $T+F \stackrel{\delta_{t f}}{\longrightarrow} F$ & $I_{5} \stackrel{\sigma_{2}}{\longrightarrow} I_{5}+Z_{5}$ & $I_{5} \stackrel{\pi}{\rightarrow} V_{5} \times 200$ \\
$U+F \stackrel{\delta_{u f}}{\longrightarrow} F$ & $T+V_{5} \stackrel{\beta_{R 5}}{\longrightarrow} I_{5}$ & $I_{4} \stackrel{\sigma_{2}}{\longrightarrow} I_{4}+Z_{4}$ & $I_{4} \stackrel{\pi}{\rightarrow} V_{4} \times 200$ \\
$U \stackrel{\delta_{u t}}{\longrightarrow} T$ & $T+V_{4} \stackrel{\beta_{X 4}}{\longrightarrow} I_{4}$ & $I_{5}+Z_{5} \stackrel{\sigma_{1}}{\longrightarrow} U+I_{5}+Z_{5}$ & $V_{4} \stackrel{\sigma_{3}}{\longrightarrow} F+V_{4}$ \\
$U \stackrel{\delta_{u z}}{\longrightarrow} Z_{4}$ & $I_{4}+Z_{4} \stackrel{\sigma_{1}}{\longrightarrow} U+I_{4}+Z_{4}$ & $I_{5} \stackrel{\delta_{i}}{\longrightarrow} 0$ & $V_{4} \stackrel{\delta_{v}}{\longrightarrow} 0$ \\
$U \stackrel{\delta_{u z}}{\longrightarrow} Z_{5}$ & $I_{5}+Z_{5} \stackrel{\delta_{i z}}{\longrightarrow} Z_{5}$ & $I_{4} \stackrel{\delta_{i}}{\longrightarrow} 0$ & $V_{5} \stackrel{\delta_{v}}{\longrightarrow} 0$ \\
$T \stackrel{\delta_{t}}{\longrightarrow} 0$ & $Z_{5} \stackrel{\delta_{z}}{\longrightarrow} 0$ & $I_{4}+Z_{4} \stackrel{\delta_{i z}}{\longrightarrow} Z_{4}$ & \\
& $Z_{4} \stackrel{\delta_{z}}{\longrightarrow} 0$ & $V_{5} \stackrel{\mu}{\rightarrow} V_{4}$ & \\
\hline
\end{tabular}

Table 2 Reaction-centric view of the HIV model.

Most of the parameters were available in literature in the suitable formats, as also used in the deterministic model, while others have required some tuning against the known macroscopic behaviour of the model, because they were unknown or not precisely defined. For instance, the $\delta_{v}$ rate relative to virus clearance and expected to be relative to a period of 2-3 days, has resulted in being a very influential parameter for the overall infective behaviour: the slower $\delta_{v}$, the more difficult for the infection to develop.

We have constructed the stochastic model of the HIV infection by first turning the equations in Figure 6 into a reaction centric description, shown in Table 2. From this description a population-based representation can easily be derived by specifying the behaviour of each involved agent. For instance, the behaviour of $U$ is given in terms of all the reactions to which $U$ participates, which appears in the first column of the table. This description is given in the specification language of the PRISM model checker we have used (see Section 3.3).

Figure 3(a, c, e) reports the results of a stochastic simulation of the model. The inherent noise, i.e. fluctuation of quantities, and its amount can clearly be appreciated, especially when comparing these results with the averaged results of the deterministic model.

Embedding HAART therapy in the stochastic model. We have modelled the effects of the anti-HIV therapies within our stochastic model, according to the representation of this therapy described by the equations of the deterministic model (see page 7). Here, a notion of time flow is essential to express the degradation of the efficacy of the therapy during the treatment period. This has been modelled by adding a parameter that downgrades the infectivity of the virus, as prescribed by the deterministic equations. This parameter is a multiplicative coefficient of the rates ruling infective reactions and those regarding viral replication. This coefficient varies in time, starting from a $60 \%$ reduction of these reactions and degrading to a null effect over a time span of 400 days. These settings have been obtained by tuning the model on the macroscopic expected behaviour of the system. For instance, it has been observed that higher initial values of the reduction coefficient would have led to the unrealistic complete eradication of the virus. Figure 3(b, $\mathrm{d}, \mathrm{f})$ reports the time course of the viral infection in the presence of a therapy treatment from about day 200 and lasting about 400 days. The time values are slightly approximated since the time representation within the model is also based 

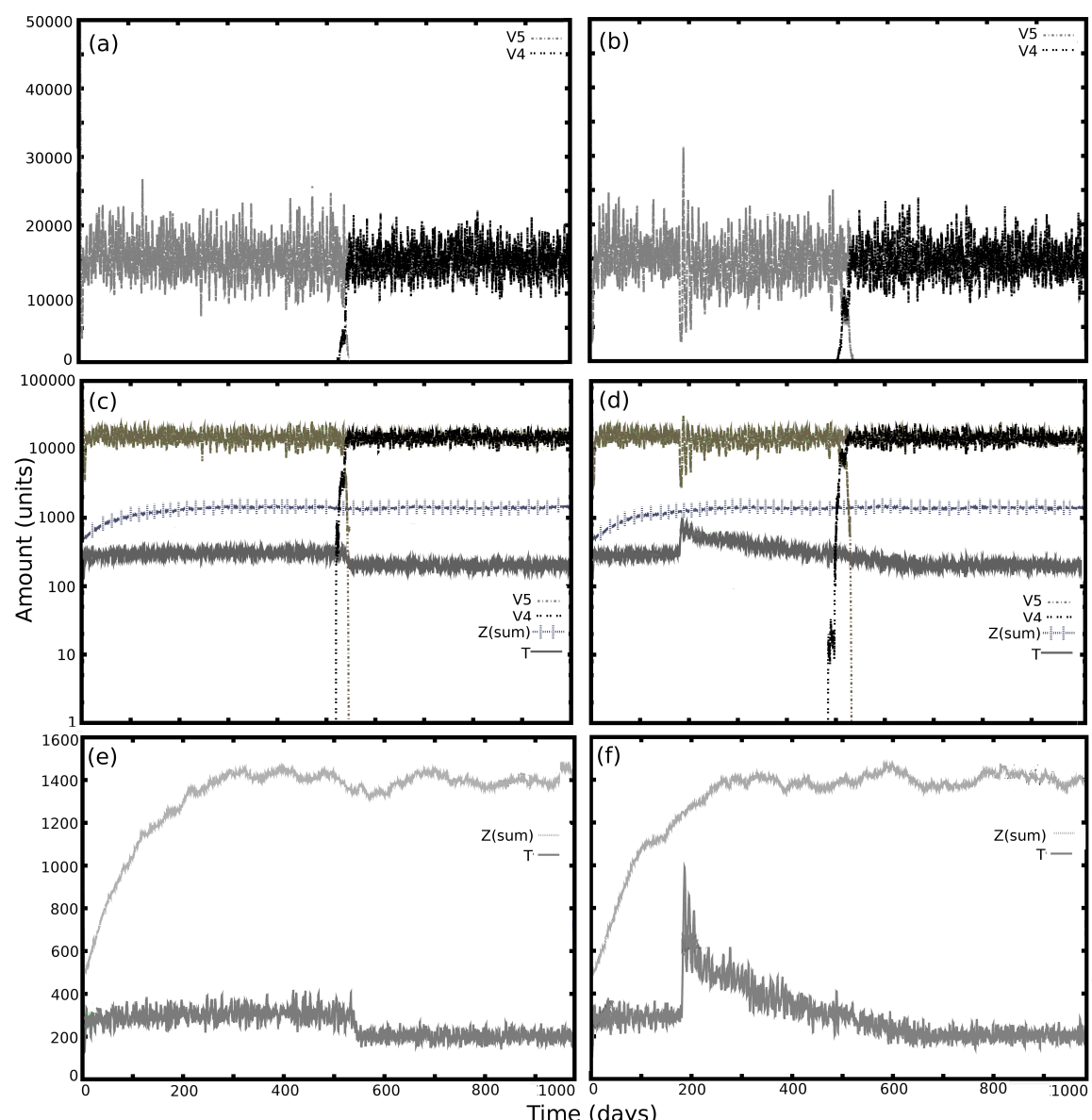

Fig. 3 Reduced Model: a single stochastic simulation over the [1-1000] days interval. Plot (a) illustrates the overall infective dynamics. The viral load develops initially based on a V5 population (light grey). At about day 500 the mutation occurs, V4 (black) prevails, and the system is locally perturbed. Plot (c) highlights the flattened values relative to the immune system. Here, the graphs about the viral load are reported together with those relative to Z (i.e., the sum of $\mathrm{Z} 4$ and $\mathrm{Z} 5$ ) and $\mathrm{T}$ in a log-scale. It can be seen that the $\mathrm{T}$ cell population reduces at the time of mutation, following the switch in the virus population. This is even more clearly evident in plot (e), which reports only data about Z5, Z4 and T. Here, the degradation of $\mathrm{T}$ below the 200 threshold in the later stages of the disease is evident. Figures on the right (b, $d$ and $\mathrm{f}$ ) represent stochastic simulation in the presence of HAART treatment during the approximative interval $[200,600]$ days. The plot (b) illustrates the overall infective dynamics. A perturbation in terms of an oscillation can be observed in the viral load time course in correspondence of the start of the treatment (compare with the analogous picture in plot (a) in the left side). The situation is clearer in the log-scale plot (d): T is sustained by the treatment. The increase follows the efficiency time course of the treatment. Importantly during the treatment the threshold safety level of T, less than about 200, seems less easily reachable. However, the level of $\mathrm{T}$ degrades after the suspension of the treatment and particularly, as expected, when the mutation from V5 to V4 occurs. This is also clearly visible in the plot (f). Initial conditions for these experiments are: $\mathrm{U}=200, \mathrm{~T}=1000, \mathrm{Z} 5=250, \mathrm{Z} 4=250$, and V5 $=100$ (Weinberger et al., 2009). The reference model of typical course of HIV infection can be found in (Pantaleo et al., 1993; Perelson et al., 1996; Wodarz and Nowak, 2002). 
on a stochastic phenomenon which introduces some indeterminacy. However this approximation is acceptable for our aims here.

The comparison of the results of the stochastic simulations with and without treatment (Figure 3(a, c, e) and Figure 3(b, d, f), respectively) shows clearly remarkable differences in the viral load and immune system time courses, demonstrating the effects of the therapy. In the next section we will formally assess these differences by evaluating probability values of the infection being in a certain state, amongst other things. This kind of data can be elaborated by means of an automated procedure (PRI). We believe that this kind of quantitative, formal and automated analysis may represent a step ahead in the understanding of the modelling efforts of infection development, by shifting the attention from an informative, but empirical, analysis of the graphs produced by simulations towards more precise quantitative interpretations.

\subsection{Model checking}

Many models describing the behaviour of dynamical systems, like distributed and concurrent computational systems, have been equipped with a formal semantics that precisely describes the possible evolution of the system. Often, such a semantics is given in terms of a Transition System that defines the possible states of the modelled systems and the modalities to move from one state to another. In the last decade several formal methods, e.g. Pi-calculus and $\mathrm{P}$ systems, have been used in the simulation of biological networks. The Pi-calculus has been used for modelling biochemical systems, with molecules and their domains represented by computational processes, and reactions by communication and channel passing. The Pi-calculus offers the ability to reconfigure communication, thus it is particularly suitable for systems in which the communication network evolves in time (Regev and Shapiro, 2002).

Suitable logical formulas can represent sets of states which fulfil properties of interest. Informally speaking, a formula like

$$
\diamond a . \phi
$$

can be used to represent the set of states in which an " $a$-kind-of" transition can occur and the system evolves into a state fulfilling, in turn, the formula $\phi$ (i.e, the - . - operator is read as a temporal sequencing operator). In a sense, the states of the semantics become the model of the formulas. The problem of the verification of a formula against a state semantics is known as Model checking (Clarke et al., 1999). Recently model checking methods have been introduced in the analysis of genetic networks. Model checking is perhaps one of the most established "proof" techniques in symbolic reasoning. The concept of proof gets instantiated appropriately through an automatic search for a property to hold or not over a finite set of states. The outcome of Model checking is either an affirmative answer or a counter example, e.g. a set of states representing possible evolutions of the system that do not fulfill the formula.

Logics (Aziz et al., 2000) and model checking procedures (Baier et al., 2003) have been defined for probabilistic state semantics, that is Probabilistic model checking. A typical logic may express formulas like

$$
P>p \quad[\phi]
$$


where $\phi$ is a path formula representing a set of traces with given features, e.g. $x=0 \quad U \quad x>2$ represents the traces in which the value of the variable $x$ is constantly 0 until it becomes bigger than 2 . Overall, the above formula represents all the traces for which $\phi$ holds with probability bigger than $p$. Other examples of operators for $\phi$ are $F$, e.g. $F>=t \quad x>2$, the traces in which eventually $x>2$ after $t$ time units, and $G$, e.g. $G x<10$, globally, for all the traces, the value of $x$ is less than 10 .

We have adopted the open-source PRISM probabilistic model checker (PRI), one of the reference existing model checkers for the analysis of systems which exhibit random or probabilistic behaviour. However, our framework is not determined by the features of a specific tool, and other similar platforms could have been chosen. PRISM models are specified in a formal language that describes the entities present in the model, their behaviour and their quantities. In our settings, the entities are $T$ cells, $R 5$ and $X_{4}$ Virus strains, etc. One or more entities can participate synchronously to an event that causes a state change. The state typically consists of the values of the variables of the model. For instance, $T$ cell infection is an event to which virus strains and $T$ cell participate. This event leads to an increased number of infected cells, and less $T$ cells and viruses, which have turned together into the infected cells. Participation to events is realised by executing suitable actions. Actions have stochastic rates associated. Variables keep trace of the amount and variations of involved entities. Such quantities affect the stochastic dynamics as expected, since dynamics is ruled by the law of mass action, as standard (according to this law, the strength of a reaction, its probability to occur quickly say, depends on the associated rates and the amount of entities ready to participate into the reaction).

Figure 4 shows the PRISM code for $T$ cell. The variable $t$ keeps trace of the amount of $T$ cell, and has a limited range since the model has to be finite. Then, several possible actions, corresponding to the participation to reactions, are defined. Each consists of a name, an integrity constraint, a rate and an effect. [five] corresponds to the reaction $T+V_{5} \stackrel{\beta_{R 5}}{\longrightarrow} I_{5}$, i.e. the infection of a $T$ cell by a $R 5$ strain virus $V_{5}$. The constraint requires the existence of a $T$ cell to be infected. The rates embeds the contribution of the amount of $T$ cells ( $t)$, the machinery that reflects strain mutation into reactions (beta - $(\mathrm{Kr} 4 * \mathrm{f}))$ ) and the representation of the therapy $(1-((100.0-$ thpy $) / 100))$, with thpy varying according to the effectiveness of the therapy in time. The effect of [five] is to decrease the number of $T$. Correspondingly, [five] is also a reaction to which $V 5$ and $I 5$ participate, as prescribed in the respective code. It is worth noting how rates are defined so as to respect stochastic semantics. Rates of synchronously participated reactions, such as [five], are multiplied to determine the overall rate of the corresponding transition (i.e. the parameter of the associated negative exponential distribution).

\section{Results}

\subsection{Sensitivity Analysis}

To gain some understanding of dependence of the solution on the parameters, we calculate the sensitivity of the key parameters, which is the sensitivity of dependence of the dependent variable say $y$ upon changes in the independent variable, 


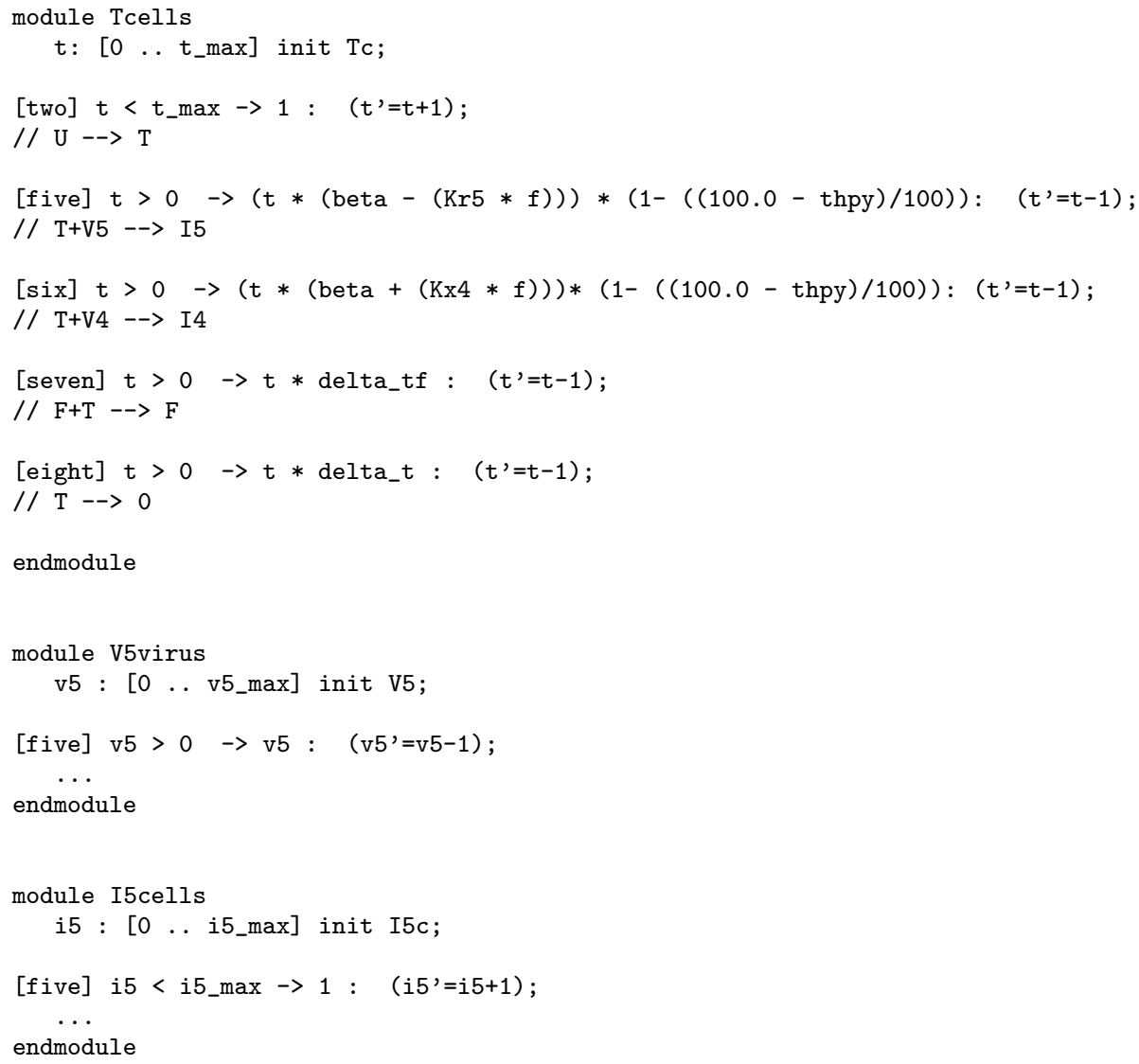

Fig. 4 The PRISM code: excerpts.

say $x$. Thus, the sensitivity value $\alpha$ is the proportional change in $y$ for a given proportional change in $x$, i.e. $\alpha=\frac{x \delta y}{y \delta x}$. A large parameter exploratory analysis using the stochastic approach may allow parameters that determine the sensitivity of the model, which is the method we have adopted. One approach to sensitivity analysis is to apply all the combinations of parameters but this approach is extremely time consuming and hence impractical for multitude parameter systems. An alternative approach, for a $N$ parameter model, is to fix $N-1$ parameters and vary $k^{t h}$ over the specific range. We focus on the parameters which can be altered by drug therapies.

\subsubsection{Dynamics of CTLs on transition of $R 5$ to $X_{4}$ switch}

The evidences suggest that CTLs response contributes to control HIV-1 infection in-vivo (Borrow et al., 1997; Carrington and OBrien, 2003; Jin et al., 1999; Kalams et al., 1999; Phillips et al., 1991). However, disease progression rate to AIDS varies person to person which indicates that CTLs response plays a protective role in progression. Here, we simulate CTLs response during HIV-1 superinfection and 
R5 to X4 phenotype switch. We have varied parameter $\delta_{u z}$ (proliferation of CTLs from immature $\mathrm{T}$ cells) to analyse the effect of CTLs and infected cells without varying other parameters related to CTLs.
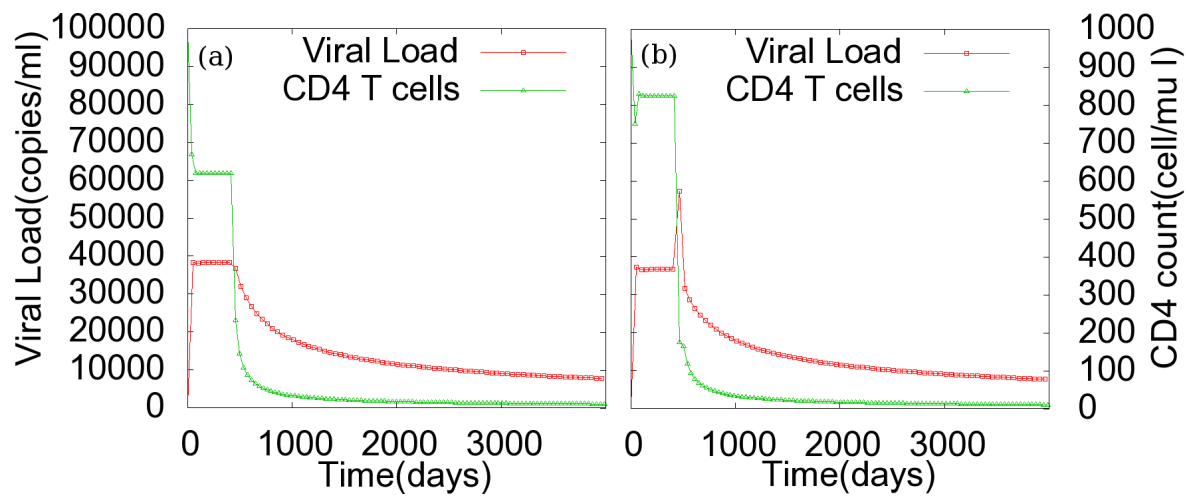

Fig. 5 Effect of immune response on the model due to $\delta_{u z}$ parameter: plot (a) $\delta_{u z}=0.005$, where CD4+ T cells are at around 600 while in (b) $\delta_{u z}=0.01$, with boosted immune response, CD4+ T cells are more than 800 .

In Figure 5, we varied parameter $\delta_{u z}$ to observe the dynamics of viruses and $\mathrm{CD} 4+\mathrm{T}$ cells. CD4 $\mathrm{T}$ cells decrease in the presence of weaker immune system $\left(\delta_{u z}=0.005\right)$, while increasing the proliferation rate of CTLs from immature cells $\left(\delta_{u z}=0.01\right)$ increases $\mathrm{CD} 4+\mathrm{T}$ cells, but do not make much difference in the appearance of X4 phenotype (see Figure 5). The simulation results suggest that boosting immune response might help to increase CD4+ T cells during initial stage of infection. The drug which boosts CTLs response might help in controlling HIV-1.

\subsubsection{Production rate of TNF}

The interaction of cytokine receptors provides essential immune and inflammatory responses hence plays a major role in the immune response to pathogens. In addition, cytokines such as TNF- $\alpha$ are strongly correlated with replication of HIV (Matsuyama et al., 1991). However, finding a balanced dose of TNF inhibitor therapy might be useful to reduce HIV replication. Here, we have varied the production rate of TNF (parameter $\sigma_{3}$ in deterministic model) to observe the effect of CD4+ T cells, CTLs cells and viral particles. The results are in Figure 6.

Figure 6 shows dynamics of CD4+ T, CTLs cells and Viral load by varying production rate of TNF. It might be possible to apply TNF inhibitor therapy upto certain level $\left(\sigma_{3}=0.001\right.$ in Figure 6$)$ so minimum level of TNF concentration remains in the system.

\subsection{HAART}

There are many different factors in HIV-1 patients that have significant implications for the generation of drug resistant viruses. HIV reverse transcribed to 

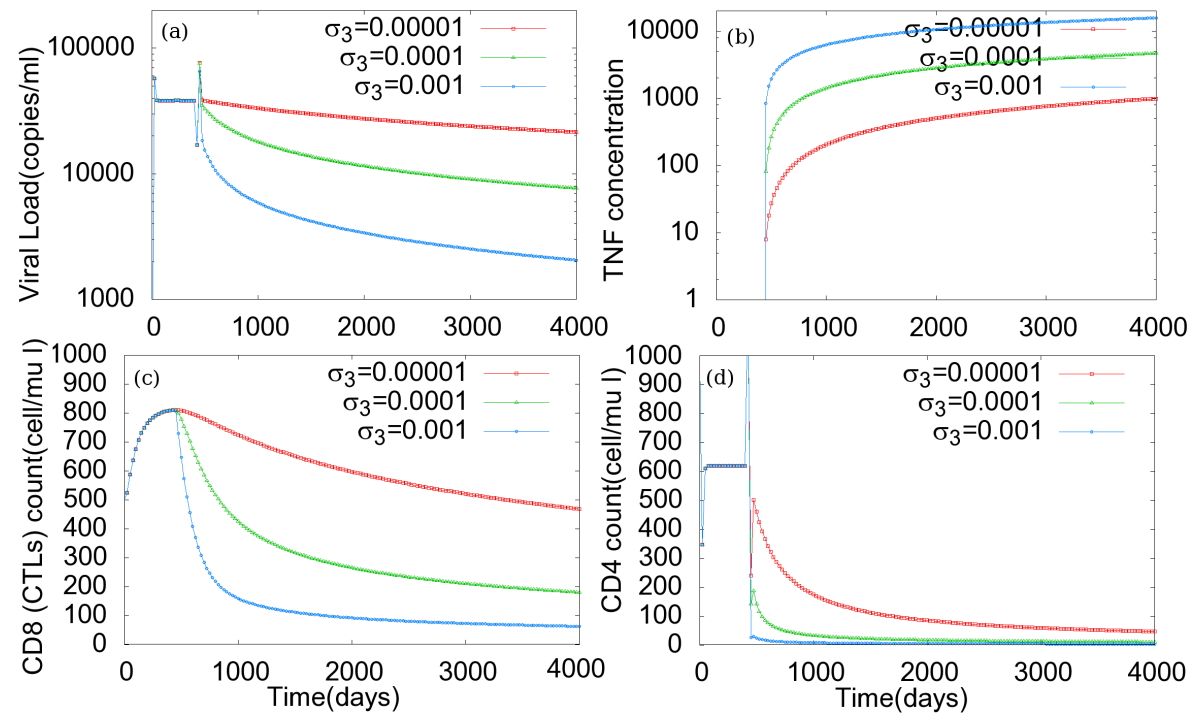

Fig. 6 Sensitivity of $\sigma_{3}$ parameter: decrease in the production rate of TNF clearly shows increase of CD4+ T and CTLs cells and decrease of Viral load. Plot (a) shows Viral dynamics (b) shows time course dynamics of TNF dynamics, plot (c) is CD4+ T cells and plot (d) is CTLs dynamics. Red $(\square)$, Green $(\triangle)$, Blue (o) lines correspond to $\sigma_{3}$ values $0.00001,0.0001$ and 0.001 respectively.

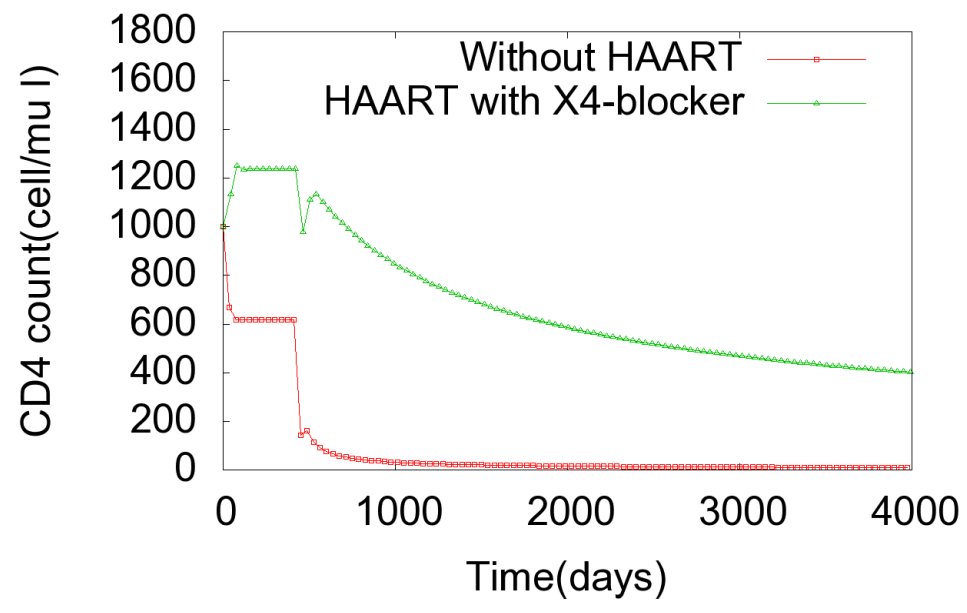

Fig. 7 Model outcome with HAART with X4 blocker and without HAART therapy: Drug treatment simulated from time $t=100$ to $t=700$. The concentration of CD4+ T cells is plotted using red line $(\square)$ and green line $(\triangle)$ denoting without drug therapy and with drug therapy respectively. Here, we have set $\eta_{R T}$ and $\eta_{P I}$ as 0.9 initially, those are decreased with time by 0.01 factor until the end of the therapy $\left(\eta_{R T}=0.99 \times \eta_{R T}\right.$ and $\left.\eta_{P I}=0.99 \times \eta_{P I}\right)$.

DNA is an error prone process and the error rate is estimated as $2.5 \times 10^{-5}$ to $3.0 \times 10^{-5}$ per base-pair replication cycle (Mansky and Temin, 1995). The effect of the therapy reduces as viruses mutate and possibly some of viral strains proliferate without any effect on therapy. We have simulated HAART therapy with 
$\mathrm{X} 4$ blocker drug combination from day $t=100$ to day $t=700$ (see Figure 7 ). We reduce the effectiveness of the drug by assuming that viruses become resistance to the drug during the course of the therapy. The simulation results suggest that HAART is more effective when it is used combined with X4 blocker drug (see Figure 7).

\subsection{Formally assessing anti-HIV therapies}

We evaluate quantitatively the effects of the HAART therapy, which we have embedded in the stochastic model as explained in Section 3.2. In doing so, we intend to highlight how relevant information contained in the model can precisely be referred and assessed. We do this by integrating in our framework stochastic model checking techniques, i.e. a formal and automated procedure for validating properties of a model. An introduction to probabilistic model checking is beyond the scope of this paper, we refer the interested reader to the cited literature and references therein. Other recent works are (Fisher et al., 2007; Fromentin et al., 2007; Mateus et al., 2007). We introduce informally the main concepts by examples.

\subsubsection{Therapy effect formalisation}

Comparing the quantitative results of the stochastic simulations in the form of Figure 3(a, c, e) and Figure 3(b, d, f), one can have an idea about the efficacy of the therapy. For instance, it appears quite evident that the therapy actually has an impact on sustaining $T$ levels as long as the treatment is provided and especially in the initial phases, where its effectiveness is bigger, see Figure 3(b). This also has an effect on the amount of $V 5$ viral load, see Figure 3(f). This kind of observation relies on the capability of properly interpreting the graphs, typical of a domain expert.

We move from such a correct and informative, but somehow empirical, interpretation of data to a more precise and formal approach by specifying properties of interest as logical formulae. This has the advantage of requiring a definition of all the aspects of interest, of referring to a formal unambiguous semantics, and of having an automated procedure for verifying the formulae.

We discuss two properties of interest:

1. We take the number of infected cells leading to virus replication as a measure of the spread of infection. Namely, this can be obtained by counting the number of times that either action causing viral replication:

$$
I \stackrel{\pi}{\rightarrow} 100 \times V 5 \quad I \stackrel{\pi}{\rightarrow} 200 \times V 4
$$

is applied. Technically this can be done by using a reward structure supported by PRISM: values are associated with states or transitions of the model. Rewards allow us to take quantitative measures of the model behaviour. In our case, we simply take a counter of rule application, $i_{-} t o \_v$. Then we can compute the cumulative value of the counter, Reward operator, within a given time interval, here $[200.0,600.0]$ :

$$
R\left\{" i_{-} t o \_v "\right\}=? \quad[C<=200.0] \quad \text { and } \quad R\left\{" i_{-} t o \_v "\right\}=? \quad[C<=600.0]
$$


2. We consider the amount of healthy $T$ cell $t$ as a measure of resistance to the virus attack. We are interested in the probability that $t$ is less than the threshold 200.0, which is considered as a marker for HIV infection, in a time interval subsequent to the beginning of virus attack, [200.0,600.0] again:

$$
P=? \quad[F[200.0,600.0] t<=200.0]
$$

It is worth noting also the methodological relevance of the formal specification of properties, where one has to declare, and hence carefully consider, all the facets of the problem. It is clear that the behaviour of complex systems change in time, but the formal specification helps make the interval of interest explicit.

\subsubsection{Results of the computational analysis}

PRISM supports two ways of giving semantics to the properties of the model. The first one is by constructing the complete CTMC relative to the model. In settings like those we are discussing, involving thousands of entities which combinatorially may generate state explosion, this can be particularly costly and become unfeasible. The second one is an approximated verification of probabilistic formulas based on sampling a certain number of traces in order to determine probability values and formula validity. Given the dimensions of our model, we have adopted the second option.

PRISM allows the degree of approximation to be controlled by means of two parameters; precision and confidence. A sufficiently high number of traces are sampled so that the confidence is an upper bound to the probability that the approximation error is bigger than the precision. Also the max length of sampled paths can be set. We have used

approx $=0.08$ conf $=0.8$ num samples $=249$ max path length $=50000000$

Given the exploratory nature of our paper, we have accepted a quite high value for confidence, which however requires an analysis lasting about $48 \mathrm{~h}$ on a $2.66 \mathrm{GHz}$ Intel 4-Core i5 with $8 \mathrm{~Gb}$ RAM memory (we have run in parallel two instances of PRISM, one for each model). The results are reported in the following table:

\begin{tabular}{c|c|c} 
& Plain model & Therapy model \\
\hline \hline$R\left\{" i_{-} t o \_v "\right\}=?[200.0<=C<=600.0]$ & 50157 & 47465 \\
\hline$P=?[F[200.0,600.0] t c<=200.0]$ & $\mathbf{0 . 8 0 3 2}$ & $\mathbf{0 . 3 2 9 3}$ \\
\hline
\end{tabular}

Automated verification yields quantitative measures of the investigated properties. The above table shows that in the time interval specified the therapy strongly reduces the probability that the number of $T$ cell falls below the threshold used as a marker of infection, when the treatment period is considered. Differently, the number of infected cells remains basically constant. This highlights the need for further investigations on this phenomenon, which could have several explanations, like the difference in scale of the viral load that tends to blur minimal differences on the overall time interval considered, or a different distribution of cell infections over time. This is a topic for future work. However, these perhaps minimal variations lead anyway to a different emerging behaviour, as it can be observed in both the graphs and in the numerical assessment of probabilities. 


\section{Conclusion}

Viruses that cause infectious diseases present critical issues of public health and economic welfare. There are several strains of HIV present worldwide and often HIV patients carry multiple strains simultaneously in their body and indeed nowadays HIV is being addressed through large-scale deep genomic sequencing. During the HIV infection multiple strains of the virus arise which are able to use different chemokine coreceptors particularly CCR5 and CXCR4.

Here, we represent the model of CTLs response during evolutionary multistrain infection of HIV-1, especially transition of R5 to X4 phenotype that helps to gain better insight of the complexity of the HIV-1 infection process. We observed that CTLs response is a key aspect in HIV infection. However, viruses escape from immune system by mutation. Simulation results show different behaviors of the system by varying CTLs response parameter.

There are many options available for drug therapy in the treatment of HIV infection such as Highly Active Antiretroviral Therapy, Maraviroc and gene therapy (Zinc-finger nucleuses). We noticed, interruption and discontinuation of HAART therapy may result in early appearance of R5 to X4 phenotype switch. However according to our model, X4 strain blocker drug combined with HAART may decrease viral load and increase T cells even after R5-X4 phenotype switch (Figure 7).

Our model also represents a general framework to investigate switching dominance of strains and arising new dominant strains during different phases of therapy. The software and the models are also general frameworks which can be used by other users to incorporate different parameters (the program is freely available upon requests from the authors).

We have illustrated the potential benefits for the study of viral infections and therapy assessment of using formal methods techniques on top of quantitative models. We have done this by presenting experiments on a proof-of-concept scenario regarding HIV infection.

The main contribution of this paper is to illustrate how properties of interest for the study of viral infections can be formalised in a general purpose logic, as the one supported by PRISM, how their verification can precisely characterise the numerical results of simulations, and how this can be helpful in comparing and assessing different antiviral therapies. Another important aspect is the attempt to combine a determinist approach (important when testing nonlinearities) and a stochastic one (important when testing fluctuations) to fuel information towards a model checker. Work in progress will bring refinement in all the steps of this procedure. We intend to explore the expressiveness of other supporting tools for model checking, tailor the class of logical language for better expressing properties of interest in the specific field described, and apply the approach to realistic case studies in collaboration with experimental virology labs.

Work in progress focuses on combining in a general modelling framework the viral multi-strain surveillance due to the variability of strain behaviour and the correct identification of a medical condition which is a central element in clinical practice. The combination of "classic" modelling techniques and model checking approaches could become a solid stream of clinical bioinformatics, which is for now concerned with bringing together bioinformaticians and biostatisticians to develop methods and tools for analysis and visualisation of complex clinical and genetic patient datasets. 


\section{References}

The PRISM model checker. Technical report, Internet Engineering Task Force. http://www.prismmodelchecker.org.

S. Agrawal, C. Archer, and D.V. Schaffer. Computational Models of the Notch Network Elucidate Mechanisms of Context-dependent Signaling. PLoS Compututation Biology, 5(5):e1000390, 2009.

C.L. Althaus and R.J. De Boer. Dynamics of Immune escape during HIV/SIV Infection. PLOS Computational Biology, 4(7):e1000103, 2008.

B. Asquith, C.T.T. Edwards, M. Lipsitch, and A.R. McLean. Inefficient Cytotoxic T Lymphocyte Mediated Killing of HIV-1Infected Cells In Vivo. PLOS Biology, 4(4):e90, 2006.

A. Aziz, K. Sanwal, V. Singhal, and R. Brayton. Model checking continuous time Markov chains. ACM Transactions on Computational Logic, 1(1):162-170, 2000.

C. Baier, B. Haverkort, H. Hermanns, and J.P. Katoen. Model-checking algorithms for continuous-time Markov chains. IEEE Transaction on Software Engineering, 29(6):524-541, 2003.

P. Borrow, H. Lewicki, X. Wei, M.S. Horwitz, N. Peffer, H. Meyers, J.A. Nelson, J.E. Gairin, B.H. Hahn, M.B.A. Oldstone, and G.M. Shaw. Antiviral pressure exerted by HIV-1 specific cytotoxic T lymphocytes (CTLs) during primary infection demonstrated by rapid selection of CTL escape virus. Nature Medicine, 3:205-211, 1997.

M.J. Cannon, P.J. Openshaw, and B.A. Askonas. Cytotoxic T cells clear virus but augment lung pathology in mice infected with respiratory syncytial virus. The Journal of Experimental Medicine, 168:1163-1168, 1988.

M. Carrington and S.J. OBrien. The influence of HLA genotype on AIDS. Annual Review of Medicine, 54:535-551, 2003.

J. Casazza, M. Betts, L. Picker, and R. Koup. Decay kinetics of human immunodeficiency virus-specific CD8 $+\mathrm{T}$ cells in peripheral blood after initiation of highly active antiretroviral therapy. Journal of Virology, 75:6508-6516, 2001.

F. Celada and P.E. Seiden. Affinity maturation and hypermutation in a simulation of the humoral immune response. Journal of Immunology, 26:1350-1358, 1996.

L. Chao, M.P. Davenport, S. Forrest, and A.S. Perelson. A stochastic model of cytotoxic T cell responses. Theoretical Biology, 228:227-240, 2004.

F. Ciocchetta and J. Hillston. Bio-PEPA: a Framework for the Modelling and Analysis of Biochemical Networks. Theoretical Computer Science, 410(33):30653084, 2009.

E.M. Clarke, O. Grumberg, and D.A. Peled. Model Checking. The MIT Press, Cambridge MA, 1999.

R.J. De Boer and A.S. Perelson. Towards a general function describing T-cell proliferation. Journal of Theoritical Biology, 175:657-576, 1995.

M. Dybul, A.S. Fauci, J.G. Bartlett, J.E. Kaplan, and A.K. Pau. Panel on Clinical Practices for Treatment of HIV (September 2002): Guidelines for using antiretroviral agents among HIV-infected adults and adolescents. Annals of Internal Medicine, 137(2):381-433, 2002.

D. Finzi, J. Blankson, J.D. Siliciano, J.B. Margolick, K. Chadwick, and et al. Latent infection of CD4+ T cells provides a mechanism for lifelong persistence of HIV-1, even in patients on effective combination therapy. Nature Medicine, 5: 
512-517, 1999.

J. Fisher, N. Piterman, A. Hajnal, and T.A. Henzinger. Predictive modeling of signaling crosstalk during C. elegans vulval development. PLoS Computational Biology, 3(5):e92, 2007.

J. Fromentin, J.P. Comet, P. Le Gall, and O. Roux. Analysing gene regulatory networks by both constraint programming and model-checking. In The 29th Annual International Conference of the IEEE EMBS, pages 4595-4598. IEEE, Washington DC, United States, 2007.

H.R. Fryer, A. Scherer, A. Oxenius, R. Phillips, and A.R. McLean. No evidence for competition between cytotoxic T-lymphocyte responses in HIV-1 infection. Proceeding of Royal Society of Biosciences, 276(1677):4389-4397, 2009.

V.V. Ganusov and R.J. De Boer. Estimating Costs and Benefits of CTL Escape Mutations in SIV/HIV Infection. PLoS Computational Biology, 2(3):e24, 2005.

L. Gray, J. Sterjovski, M. Churchill, P. Ellery, N. Nasr, S.R. Lewin, S.M. Crowe, S.L. Wesselingh, A.L. Cunningham, and P.R. Gorry. Uncoupling coreceptor usage of human immunodeficiency virus type 1 (HIV-1) from macrophage tropism reveals biological properties of CCR5-restricted HIV-1 isolates from patients with acquired immunodeficiency syndrome. Virology, 337:384-398, 2005.

P. Guermonprez, J. Valladeau, L. Zitvogel, C. Thery, and S. Amigorena. Antigen Presentation and $\mathrm{T}$ cell stimulation by dendritic cells. Annual Review of Immunology, 20:621-667, 2002.

A.T. Haase. Population biology of HIV-1 infection: viral and CD4+ T cell demography and dynamics in lymphatic tissues. Annual Review of Immunology, 17: 625-656, 1999.

J.P. Herbeuval, A.W. Hardy, A. Boasso, S.A. Anderson, M.J. Dolan, M. Dy, and G.M. Shearer. Regulation of TNF-related apoptosis-inducing ligand on primary CD4+ T cells by HIV-1: Role of type I IFN-producing plasmacytoid dendritic cells. PNAS, 102:13974-13979, 2005.

D. Ho, A.U. Neumann, A.S. Perelson, W. Chen, J.M. Leonard, and M. Markowitz. Rapid turnover of plasma virions and CD4 lymphocytes in HIV-1 infection. Nature, 373:123-126, 1995.

X. Jin, D.E. Bauer, S.E. Tuttleton, S. Lewin, A. Gettie, J. Blanchard, C.E. Irwin, J.T. Safrit, J. Mittler, L. Weinberger, L.G. Kostrikis, L. Zhang, A.S. Perelson, and D.D. Ho. Dramatic rise in plasma viremia after CD8 $+\mathrm{T}$ cell depletion in simian immunodeficiency virusinfected macaques. The Journal of Experimental Medicine, 189:991-998, 1999.

S.A. Kalams, P.J. Goulder, A.K. Shea, N.G. Jones, A.K. Trocha, G.S. Ogg, and B.D. Walker. Levels of human immunodeficiency virus type 1-specific cytotoxic T-lymphocyte effector and memory responses decline after suppression of viremia with highly active antiretroviral therapy. Journal of Virology, 73:67216728, 1999.

R. Kaslow, M. Carrington, R. Apple, L. Park, A. Munoz, A.J. Saah, J.J. Goedert, C. Winkler, S.J. O'Brien, C. Rinaldo, R. Detels, W. Blattner, J. Phair, H. Erlich, and D.L. Mann. Influence of combinations of human major histocompatibility complex genes on the course of HIV-1 infection. Nature Medicine, 2:405-411, 1996.

M. Kwiatkowska, G. Norman, and D. Parker. Algorithmic Bioprocesses : Quantitative Verification Techniques for Biological Processes. A. Condon, D. Harel, J. Kok, A. Salomaa and E. Winfree (editors), Natural Computing Series, Springer, 
391-409, 2009.

S.P. Layne, J.L. Spouge, and M. Dembo. Quantifying the infectivity of human immunodeficiency virus. PNAS, 86:4644-4648, 1998.

H. Li, Y. Cao, L.R. Petzold, and D.T. Gillespie. Algorithms and Software for Stochastic Simulation of Biochemical Reacting Systems. Biotechnology Progress, 24(1):56-61, 2008.

L. Mansky and H. Temin. Lower in vivo mutation rate of human immunodeficiency virus type 1 than that predicted from the fidelity of purified reverse transcriptase. Journal of Virology, 69:5087-5094, 1995.

D. Mateus, J.P. Gallois, J.P. Comet, and P. LE Gall. Symbolic modeling of genetic regulatory networks. Journal of bioinformatics and computational biology, 5(2B): 627-640, 2007.

T. Matsuyama, N. Kobayashi, and N. Yamamoto. Cytokines and HIV infection: is AIDS a tumor necrosis factor disease? AIDS, 5(12):1405-1417, 1991.

C.A. Michie, A. McLean, C. Alcock, and P.C.L. Beverly. Lifespan of human lymphocyte subsets defined by CD45 isoforms. Nature, 360:264, 1992.

J.E. Mittler, M. Markowitz, D.D. Ho, and A.S. Perelson. Improved estimates for HIV-1 clearance rate and intracellular delay. AIDS, 13(11):1415-1417, 1999.

J.M. Murray, G. Kaufmann, A.D. Kelleher, and D.A. Cooper. A model of primary HIV infection. Mathatical Biosciences, 154(5):57-85, 1998.

G. Ogg, X. Jin, S. Bonhoeffer, P. Moss, M. Nowak, S. Monard, J.P. Segal, Y. Cao, S.L. Rowland-Jones, A. Hurley, M. Markowitz, D.D. Ho, A.J. McMichael, and D.F. Nixon. Decay kinetics of human immunodeficiency virus-specific effector cytotoxic T lymphocytes after combination antiretroviral therapy. Journal of Virology, 73:797-800, 1999.

G. Pantaleo, C. Graziosi, and A.S. Fauci. The Immunopathogenesis of Human Immunodeficiency Virus Infection. The New England Journal of Medicine, 328: 327-335, 1993.

A.S. Perelson, A.U. Neumann, M. Markowitz, J.M. Leonard, and D.D. Ho. HIV-1 dynamics in vivo: Virion clearance rate, infected cell life-span, and viral generation time. Science, 271:1582-1586, 1996.

B.N. Phillips. Reduction of HIV concentration during acute infection: independence from a specific immune response. Science, 271(5248):497-499, 1996.

R.E. Phillips, S. Rowland-Jones, D.F. Nixon, F.M. Gotch, J.P. Edwards, and et al. Human immunodeficiency virus genetic variation that can escape cytotoxic $\mathrm{T}$ cell recognition. Nature, 354:453-459, 1991.

B. Ramratnam, J.E. Mittler, L. Zhang, D. Boden, A. Hurley, F. Fang, C.A. Macken, A.S. Perelson, M. Markowitz, and D.D. Ho. The decay of the latent reservoir of replication-competent HIV-1 is inversely correlated with the extent of residual viral replication during prolonged anti-retroviral therapy. Nature Medicine, 6:82-85, 2000.

B. Ramratnam, R. Ribeiro, T. He, C. Chung, V. Simon, J. Vanderhoeven, A. Hurley, L. Zhang, A.S. Perelson, D.D. Ho, and M. Markowitz. Intensification of antiretroviral therapy accelerates the decay of the HIV-1 latent reservoir and decreases, but does not eliminate, ongoing virus replication. Journal of Acquired Immuno Deficiciency Syndrome, 35:33-37, 2004.

A. Regev and E. Shapiro. Cellular abstractions: Cells as computation. Nature, 419:343, 2002. 
R.M. Ribeiro, H. Mohri, D.D. Ho, and A.S. Perelson. Modeling deuterated glucose labeling of T-lymphocytes. Bulletin of Mathematical Biology, 64(2):385-405, 2002a.

R.M. Ribeiro, H. Mohri, D.D. Ho, and A.S. Perelson. In vivo dynamics of T cell activation, proliferation, and death in HIV-1 infection: Why are CD4+ but not CD8+ T cells depleted? PNAS, 99(24):15572-15577, 2002b.

S.R. Riddell, K.S. Watanabe, J.M. Goodrich, C.R. Li, M.E. Agha, and P.D. Greenberg. Restoration of viral immunity in immunodeficient humans by the adoptive transfer of T cell clones. Science, 257:238-241, 1992.

J. Schmitz, M. Kuroda, S. Santra, V. Sasseville, M. Simon, and et al. Control of viremia in simian immunodeficiency virus infection by CD8+ lymphocytes. Science, 283:857-860, 1999.

L. Sguanci, F. Bagnoli, and P. Lio'. Modeling HIV quasispecies evolutionary dynamics. BMC Evolutionary Biology, 7(2):S5, 2007.

J.D. Siliciano, J. Kajdas, D. Finzi, T.C. Quinn, K. Chadwick, and et al. Longterm follow-up studies confirm the stability of the latent reservoir for HIV-1 in resting CD4(+) T cells. Nature Medicine, 9:727-728, 2003.

A. Sorathiya, A. Bracciali, and P. Lio'. Formal reasoning on qualitative models of coinfection of HIV and Tuberculosis and HAART therapy. BMC Bioinformatics, 11(1):S67, 2010.

M.A. Stafford, L. Corey, Y. Cao, E.S. Daar, D.D. Ho, and A.S. Perelson. Modeling plasma virus concentration during primary HIV infection. Journal of Theoretical Biology, 203(3):285-301, 2000.

X. Wei, S.K. Ghosh, M.E. Taylor, V. A. Johnson, E.A. Emini, P. Deutsch, J.D. Lifson, S. Bonhoeffer, M.A. Nowak, B.H. Hahn, M.S. Saag, and G.M. Shaw. Viral dynamics in human immunodeficiency virus type 1 infection. Nature, 373: $117-122,1995$.

A.D. Weinberger, A.S. Perelson, R.M. Ribeiro, and L.S. Weinberger. Accelerated Immunodeficiency by Anti-CCR5 Treatment in HIV Infection. PLoS Computational Biology, 5(8):e1000467, 2009.

L. Weinberger and T. Shenk. An HIV feedback resistor: auto-regulatory circuit deactivator and noise buffer. PLoS Biology, 5:e9, 2007.

F.W. Wiegel and A.S. Perelson. Some scaling principles for the immune system. Immunology and Cell Biology, 82:127-131, 2004.

D. Wodarz and M.A. Nowak. HIV dynamics and evolution. BioEssays, 24:11781187, 2002.

L. Zhang, P.J. Dailey, T. He, A. Gettie, S. Bonhoeffer, A.S. Perelson, and D.D. Ho. Rapid Clearance of Simian Immunodeficiency Virus Particles from Plasma of Rhesus Macaques. Journal of Virology, 73(1):855-860, 1999a.

L. Zhang, B. Ramratnam, K. Tenner-Racz, Y. He, M. Vesanen, and et al. Quantifying residual HIV-1 replication in patients receiving combination antiretroviral therapy. New England Journal of Medicine, 340:1605-1613, 1999 b. 